Author Pre-Print - not for distribution

(C) ACM, 2019. This is the author's version of the work. It is posted here by permission of ACM for your personal use. Not for redistribution. The definitive version was published in Halfway to the Future '19, November 19-20, 2018, Nottingham, UK. https://doi.org/10.1145/1122445.1122456

\title{
Sketching \& Drawing as Future Inquiry in $\mathrm{HCI}$
}

\author{
Miriam Sturdee \\ m.sturdee@lancaster.ac.uk \\ Lancaster University \\ Lancaster, UK
}

\author{
Joseph Lindley \\ j.lindley@lancaster.ac.uk \\ Lancaster University \\ Lancaster, UK
}

\begin{abstract}
Creating visual imagery helps us to situate ourselves within unknown worlds, processes, make connections, and find solutions. By exploring drawn ideas for novel technologies, we can examine the implications of their place in the world. Drawing, or sketching, for future inquiry in Human Computer Interaction (HCI) can be a stand-alone investigative approach, part of a wider 'worldbuilding' in design fiction, or simply ideation around a concept. By examining instances of existing practice in $\mathrm{HCI}$, in this paper we establish recommendations and rationales for those wishing to utilise sketching and drawing within their research. We examine approaches ranging from ideation, diagramming, scenario building, comics creation and artistic representation to create a model for sketching and drawing as future inquiry for HCI. This work also reflects on the ways in which these arts can inform and elucidate research and practice in $\mathrm{HCI}$, and makes recommendations for the field, within its teaching, processes and outcomes.
\end{abstract}

\section{CCS CONCEPTS}

- Human-centered computing $\rightarrow$ Human computer interaction (HCI).

\section{KEYWORDS}

drawing, sketching, comics, scenarios, design fiction, future

\section{ACM Reference Format:}

Miriam Sturdee and Joseph Lindley. 2018. Sketching \& Drawing as Future Inquiry in HCI. In HTTF '19: ACM Symposium, November 19-20, 2018, Nottingham, UK. ACM, New York, NY, USA, 10 pages. https://doi.org/10.1145/ 1122445.1122456

Please cite this version

\section{INTRODUCTION}

Drawing is vision [50]. Hence, when simple tools like the pen and paper become a method of inquiry - through sketching our thoughts and ideas - we can develop far-reaching insights that would a prohibitively long time to design and build into systems. Human Computer Interaction (HCI) already employs a wide range of methods, whose novelty are sometimes unfamiliar to computer science. Because of this, to maximise their potential, a consolidation of how we use the "offline" creative arts, in particular the drawn image,

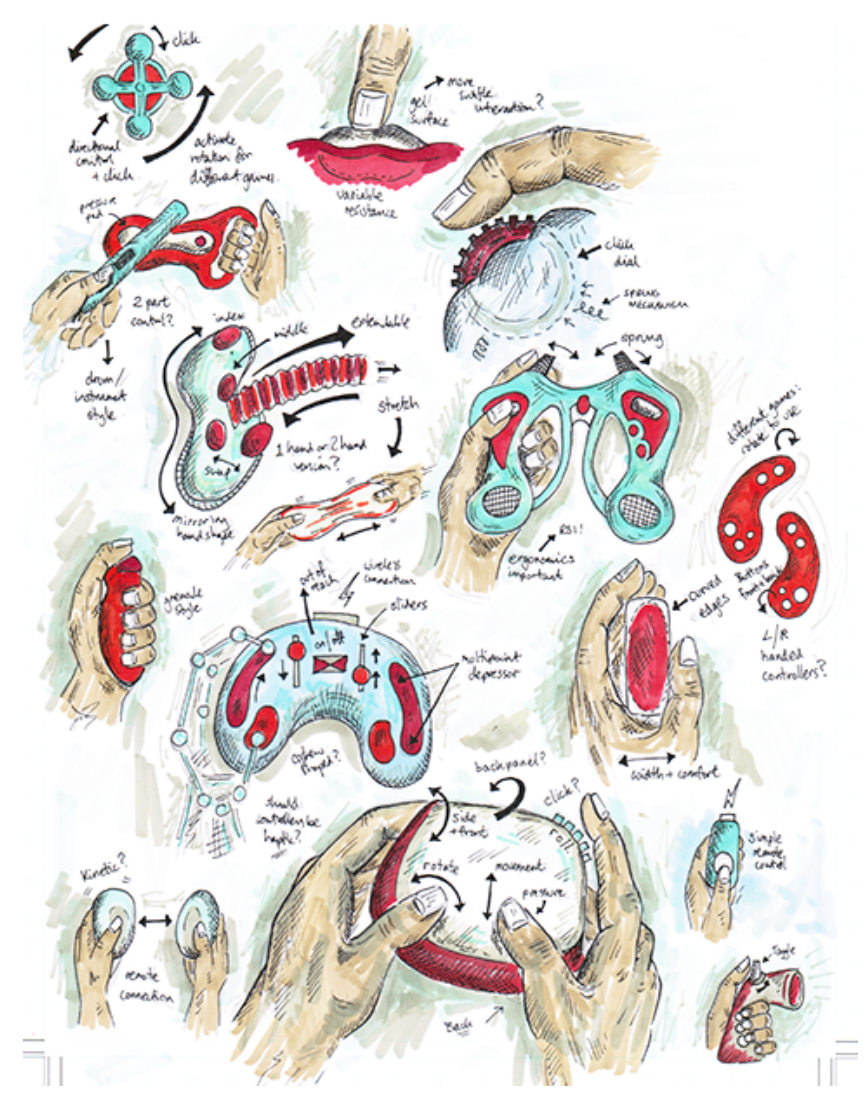

Figure 1: An example of subjective sketching and ideation of prototypes for a console game controller. The initial sketch (bottom middle) serves as a springboard for further iterations and tangents. The final image is coloured to highlight different areas and interactions. By keeping all images on the same sheet quick comparisons can be made and the story of design followed.

imports palpable benefits for the field. Drawing for the sciences is not a new concept, for example, Leonardo Da Vinci was an artist and scientist using sketching and drawing to imagine machines that were hundreds of years ahead of their time [53]. The divide between art and science is a relatively new invention and is an artificial divider. Before the professional specialisation that the Industrial Revolution demanded prised them apart, it seemed quite normal that the 'science' of nature was referred to as 'Natural Philosophy': while there is clearly "private territory" there is a lot of shared ground across art, science, research and other practices [24]. On a psychological level, the differences in creativity between great 


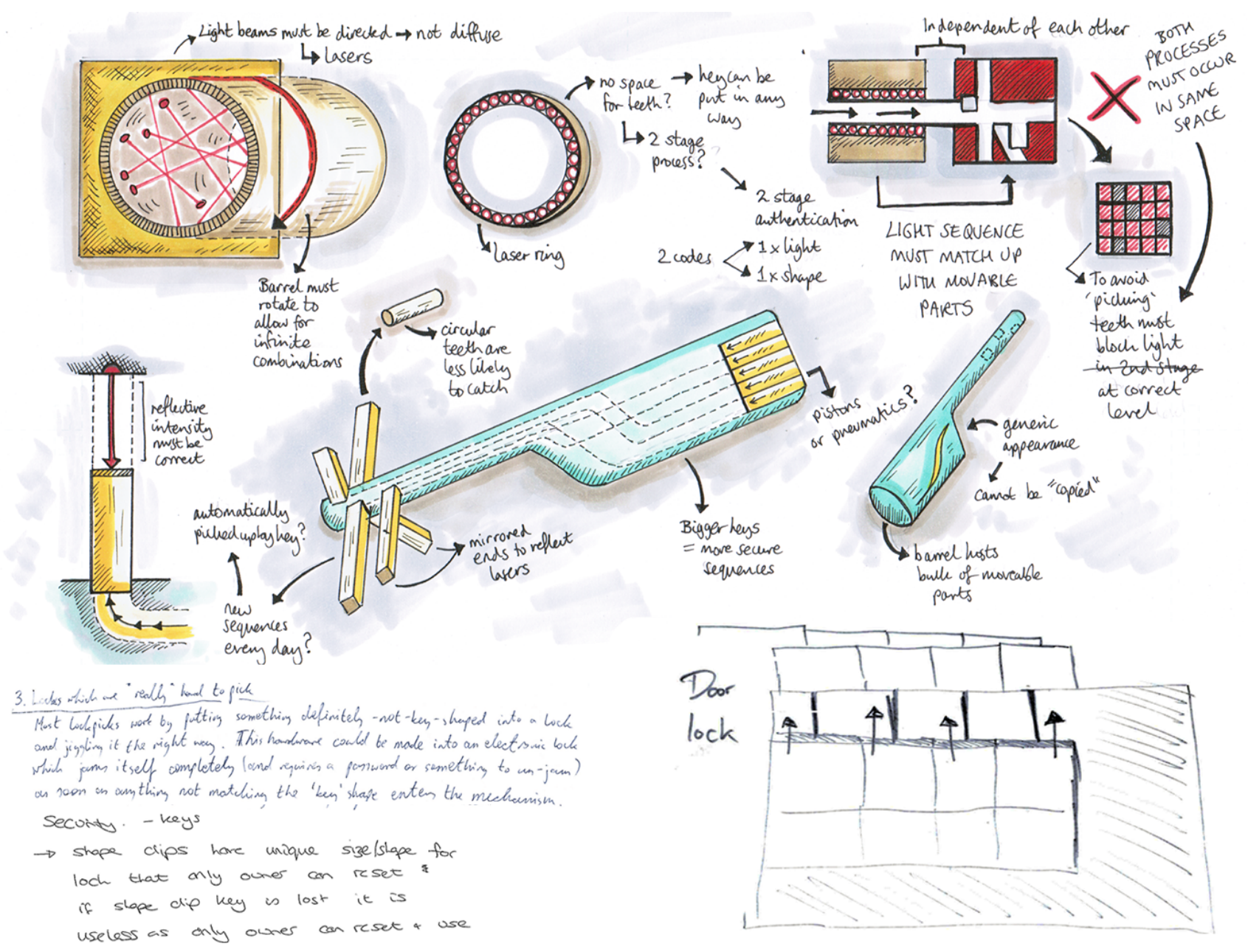

Figure 2: Participant ideation sketches and notes developed further during the research process [59]. Two participants independently sketched and made notes about the concept of a shape-changing key which could retract its shape until used (bottom). The researcher sketched a central concept, then made further sketched notes to think about the issues and practicalities associated with the design, build and use of such an object. For example, mechanical-digital keys of this nature would require many working parts, could become bulky, and would need charging.

artists and scientists are slim to none [2]. Hence, given there should be no stigma attached to approaches utilising the creative idiom, and the imagination and future-focus of the HCI field, the field owes itself to better embrace sketching and drawing in the course of its investigations.

Current research and practice around drawing and sketching in $\mathrm{HCI}$ is astounding: we have developed near-realistic paper-style interfaces [38], we can interact with sketches on digital whiteboards [9], we can see the story of a product's life-cycle within the sketched 'residue' left behind [73], we embrace sketching user experiences $[10,30]$, and we can even teach a novice to draw with gamified sketch-engines [74]. So why have we not embraced a return to the origin of the sketch? When used to illustrate and explore a concept the humble line - that which connects thought and the unknown has vast potential in research. Similarly, a 'humble divide' between the arts and sciences should be erased and surpassed. With this in mind, where exactly does the overlap between traditional 'handson' sketching and drawing fall within $\mathrm{HCI}$ ? Here, we provide an overview of ways in which we can - and have - used sketching and drawing as future inquiry in $\mathrm{HCI}$. Using these examples of creative HCI scholarship we make recommendations for the utilisation of sketching practice for making, building and understanding the nature of our emerging interactions future with technology.

This text is intended as provocation, a call to take up arms in the form of pens, pencils, and paper, and return to the roots of creative process and inquiry. To sketch and draw out one's thoughts and ideas is to communicate, to communicate creates collaboration, and to collaborate and further, to research and build, is key to our field. Here, we examine the current state of the art within the field of $\mathrm{HCI}$, and make recommendations for the field as a whole 
to embrace drawing and sketching as a form of future inquiry, a base on which to build ideas, products and impactful activity. Alongside the text, we include images which illustrate the related work and recommendations for practice, these rich, expressive research artefacts are of equal value to the written word [4, 59], and form part of the story in this paper - the enhanced captions allow snapshots into these case studies.

\section{EXISTING PROCESSES \& PRACTICE}

Sketching and drawing can be seen as subjective arts practice but it is also more than that. When a designer or HCI practitioner sketches, they are telling the future, a version of something that has the potential to be built and adopted into everyday life. Socially and culturally, we are exposed to a visual world of science fiction, in comics, books, and art, populated by robots, superheroes and fantastical technologies - and this is mirrored by HCI research. At its core, sketching can be seen as speculative, a piece of human imagination, or subjective view of the world with the potential for re-interpretation. When furthered, these sketches consolidate into drawing - the neater, more final version of the initial idea.

So far, within HCI, both sketches and these neater, more polished drawn images have been used for story-telling [57], world-building [61], ideation and elaboration [67], connection with participants and stakeholders [41, 66], objects of analysis [62] ... to name but a few examples. Sketching is a crystal ball from which new products spawn, such as Alessi's fuicy Salif lemon squeezer, which started life as a gathering of lines on a napkin [1]. In contrast, for products, programs and their adoption in HCI, we strive to recreate the simple pleasure and interaction of the pencil and paper in tablets and tabletop surfaces [37,38], or look at ways in which sketch interaction can be used for search [11], passwords [55] or learning [68]. The following text contains descriptions of hand-drawn images and their application to HCI research, in the areas of Ideation \& Communication, Teaching \& Community, Design Fiction, Comics, Storyboards \& Scenarios and Diagrams, Analysis \& Requirements. These studies set the scene for recommendations on sketching and drawing for future inquiry in Human Computer Interaction.

\subsection{Ideation \& Communication Activities}

Sketching for ideation is a common tool in the designers' repertoire [20, 30, 39], with significant overlap into HCI as a whole. Sketching in this respect makes reference to ambiguity [26] for the ideation sketch is intended as a queryable structure - because of its nonfinal state it can be edited and discussed. Sketched ideation can also be participatory [17], involve working with novices and the public $[7,63]$, or be captured to inform the design of new software [58]. Ideation from the participant approach can then further be elaborated upon to create meaningful research inquiry [63]. Rarely do projects not contain an ideation stage, although sketching for ideation is in competition or collaboration with conversational and written elements, and can be eschewed in favour of these techniques due to the fear of I can't draw [14], or lack of perceived value. Sketching for ideation communicates concepts for development (Figures 1,2), but also can be used a method of summary and documentary, as is the case of sketchnotes [52, 66]. Further, sketching purely for communicative purposes can offer additional

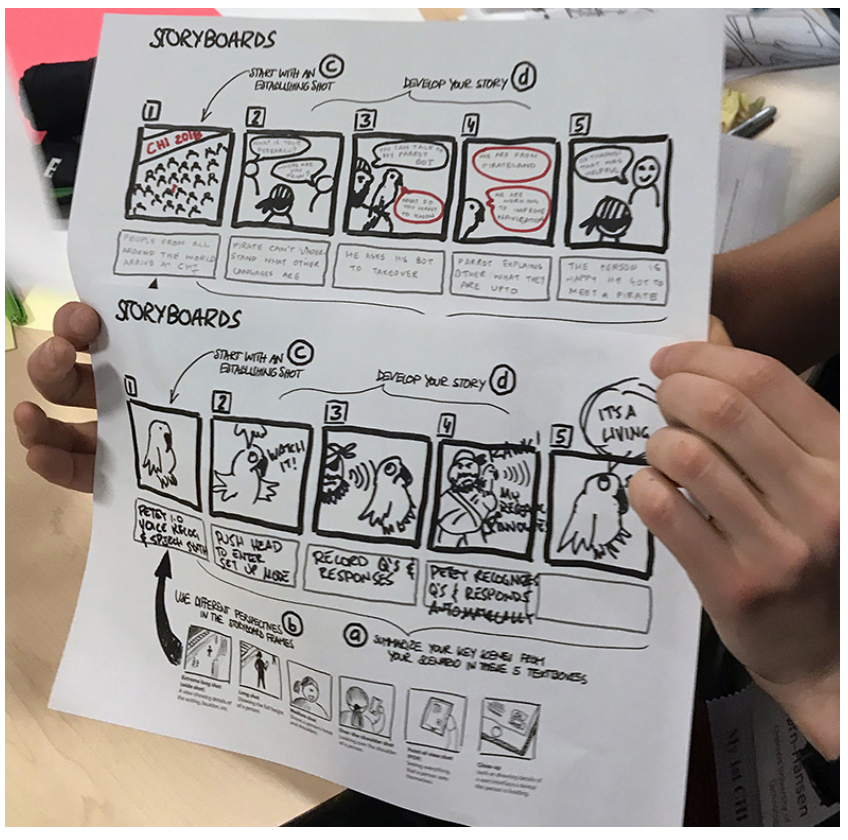

Figure 3: Example of a scenario from the HCI improv game created during the Applied Sketching in HCI course [43] [64]. Here, participants respond to verbal prompts from the audience and are challenged to create a diagram and scenario outlining their new technology, before presenting their concept and visuals to the room.

insights into both desired outcomes and the thoughts and feelings of a participant or co-worker [25], hence sketching for ideation and subsequent communication communicates intent for future work.

\subsection{Teaching \& Community}

To get by the "fear" of sketching and drawing for HCI, for the past 7 years there have been courses addressing penmanship at high profile conferences, as well as smaller, private group classes in industry. These courses often cover the absolute basics - "Learn to sketch - even if you can't draw" - [22], or particular domains such as sketchnoting $[52,66]$. More recently, combined approaches covering both practical and novel aspects have been rising in popularity, for example, hands-on Applied sketching in HCI [43, 44]. Such courses are highly subscribed, indicating demand from novices in the field, and participants often go on to use their new-found skills in their everyday practice, such as developing scenarios (Figure 3). This community is further supported by existing researchers who already utilise sketching and drawing in their forward-thinking HCI practice and host workshops [32, 42], special interest groups $[45,46]$ and offer formats for collaboration [65]. The ongoing upskilling, meetings between practitioners, and published work also trickles down into teaching at the higher education level, with researchers using sketching and ideation as a way of getting students interested in HCI [76]. 


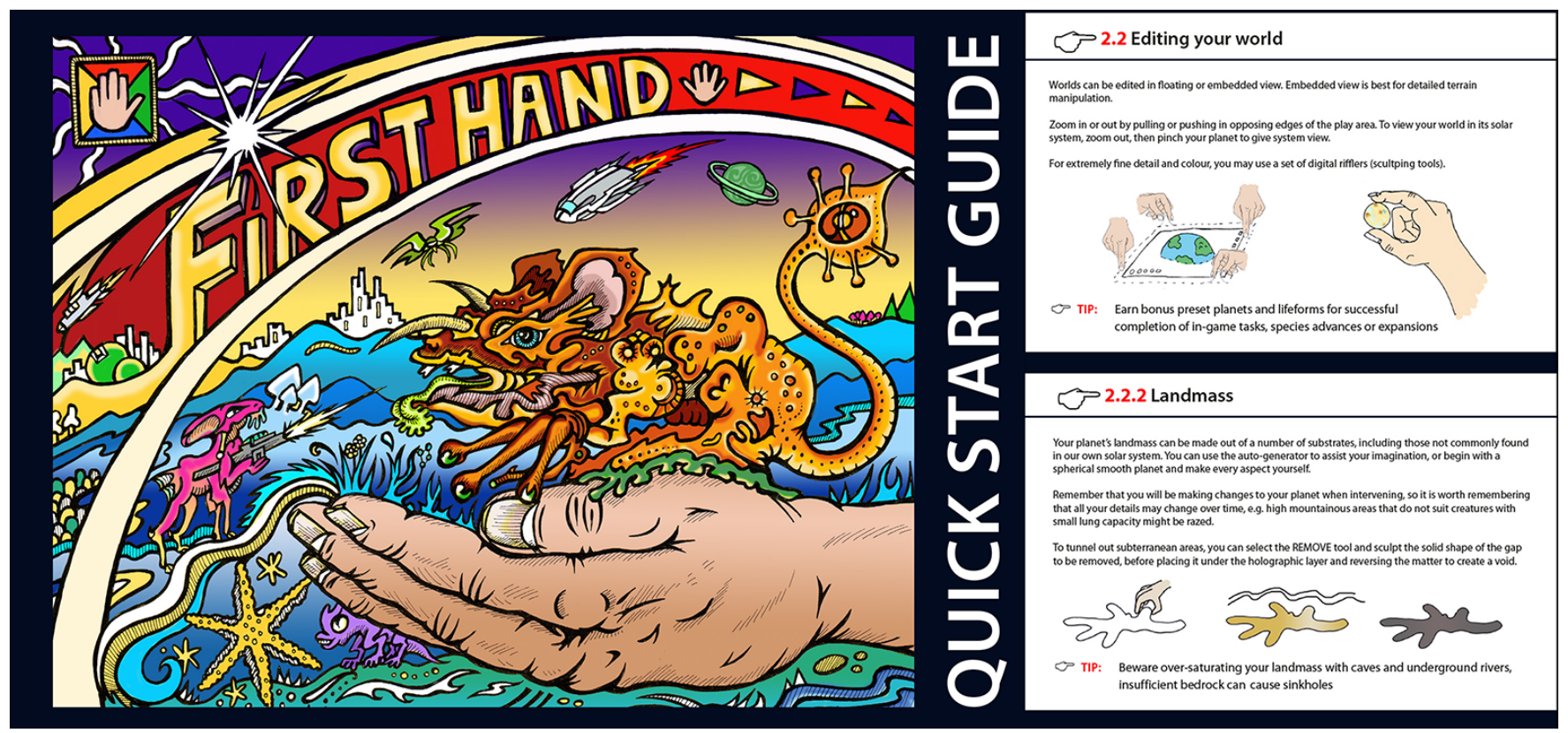

Figure 4: Sketches and elaborative drawing used to show how a future console game might work within the context of a game manual for First Hand [60]. This game is based in the research space of tangible, Augmented Reality, and shape-changing prototypes, where the user has the option to terraform or evolve species in a 'hands-on' manner. The diagrammatic sketches from the interior of the manual are in contrast to the cover image which is rich and colourful, designed as a 'plausible' design fiction which is both retro and futuristic at the same time. This work provides a rich resource for future game designers.

\subsection{Design Fiction}

Design fiction is often built around narratives [5], and can tell us about products, scenarios and worlds which do not yet exist [49]. The drawn image can be a pivotal part of a Design Fiction's worldbuilding. Working hand-in-hand with other 'entry points' into a Design Fiction, the universal visual language can lend vision to the written word, and can act as a rapidly developed prototypes for more complex entry points such as video and physical builds [15] Blythe et al. state that "making things imageable is at the heart of what designers do, from sketching to prototyping" [6] but as we have already postulated, sketching is not just for designers, and can be learned [14, 30]. Grand et al. go further than this, and suggest that sketching is "part of the design fiction toolbox" [29]. Figure 4 shows a design fiction game manual where both polished drawing and rough sketches are used to communicate the potential for a future game engine and console based on tangible and mixed reality interactions [60]. Further to this concept, Johnson et al. [36] see science fiction protoyping as also containing the use of drawn comics as a form of development. There is significant overlap between the comic as storytelling for the future, and other forms of storyboards and scenarios in HCI - but the drawn image in any of these forms remains a valuable part of the HCI process [67].

\subsection{Comics, Storyboards \& Scenarios}

How to Build a Voight-Kampff Machine is a comics based approach to design fiction that builds on the 'world building hypothesis' [15], telling the story of the concept of a digital empathy detector in a near future dating scenario (Figure 5) [61]. HCI has also embraced comics in other research contexts aside from design fiction however, such as describing ongoing work with technology and research through design [21], provocations [59] and data-driven storytelling [3], amongst others. HCI comics sit as both future inquiries and as delivery vehicles for ground-breaking research, and concepts we often find in research papers and symposiums are even finding their way into the mainstream, creating a new audience for the forward thinking focus of our field [51]. Our work in this context reflects and enforces the importance of traditional entertainment comics from the past 50 years, such as Jack Kirby's The One Man Army Corps (OMAC) which contained ideas from computers in communication with each other across the world, drones and high power submarines which are able to cruise at 12,000 feet below the ocean surface. Works in this vein show the importance of the artistic inquiry and ideation for HCI. In contrast, research storyboards and scenarios rarely find their way into the public domain, but are no less valuable in working through concepts and problems for future spaces - for example the storyboard as a method for generating and explaining interface behaviours [40], or to create scenarios based on future work ideas from existing research prototypes (Figure 6) which can then be used to gather responses from research teams and a wider public [67].

\subsection{Diagrams, Analysis \& Requirements}

The creation of images as a technique for inquiry also lends itself to post-hoc analysis. Qualitative image analysis can give us insights as to how research can be communicated, for example, via the analysis 

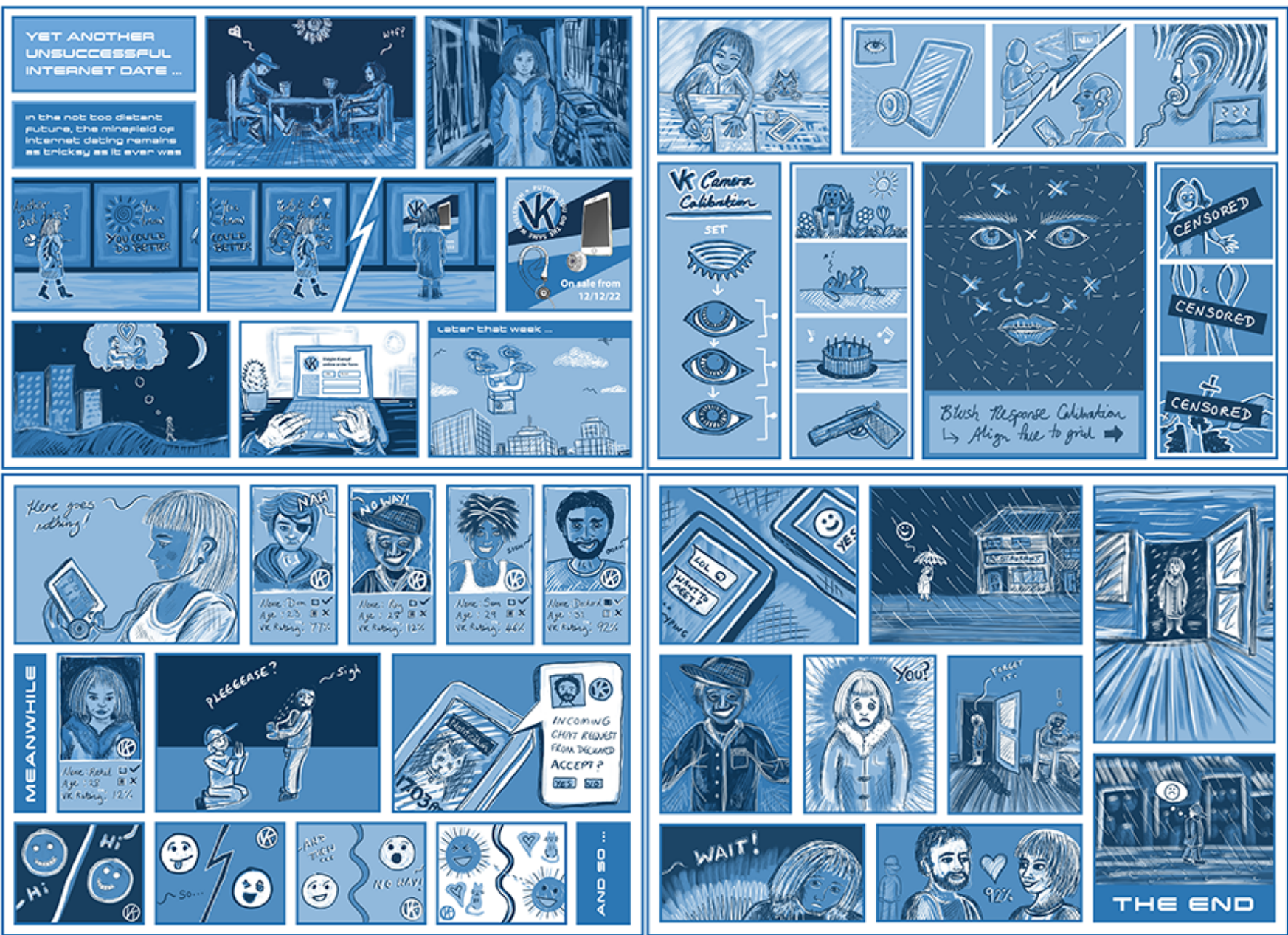

Figure 5: How to Build a Voight-Kampff Machine: Comics as future storytelling in HCI [61]. This comic investigation was based upon a series of work into digital empathy and its intersections with science fiction. The story is set in a plausible future where we add the empathy detector from the film Bladerunner to the hand-held digital device and it is supported by a dating application. The object itself is delivered by drone. The work suggests a basic story around finding love in the future, but has deeper implications for deception and trust in an increasing technological world. By making this investigation into the form of a comic, it becomes accessible to an audience which may not access research papers, but can be taken as a contribution on both sides of the academic/public divide - breaking down barriers to understanding research.

of graphical abstracts [33]), show how novices understand concepts relating to complex topics such as cybersecurity on the dark net [25], but also provide novel insights to existing processes such as software design [13]). Analysis can also provide novelty: sketching and storyboarding have been used to generate design requirements $[31,75]$. On the other side, researchers also use diagrams and UML to sketch technical processes and outcomes [12]. When used in combination, ideation, diagram sketching and storyboards or scenarios can be a powerful tool to create dialogue between participants and researchers, and have effects on future directions for sub-fields within HCI (Figure 7) [62]. In the labelled example given, the way in which the technology is represented can offer insights into how it might work in the real world setting (for example, representation of digitally rendered, physical fur), yet the image was created by a novice, with basic pen and paper for material inquiry.

\section{WAYS OF ADOPTION}

The prior examples show ways in which researchers and practitioners are already utilising sketching and drawing within the context of $\mathrm{HCI}$ inquiry, but the practice is far from universal in the field, despite the proven benefits of this form of inquiry. Sketches are a quick and low cost method of producing meaningful results, and their ambiguity allows conversation and misinterpretation before more final drawn outputs are created [26], which have their own value in storytelling and analysis. Sketching and drawing can tell 

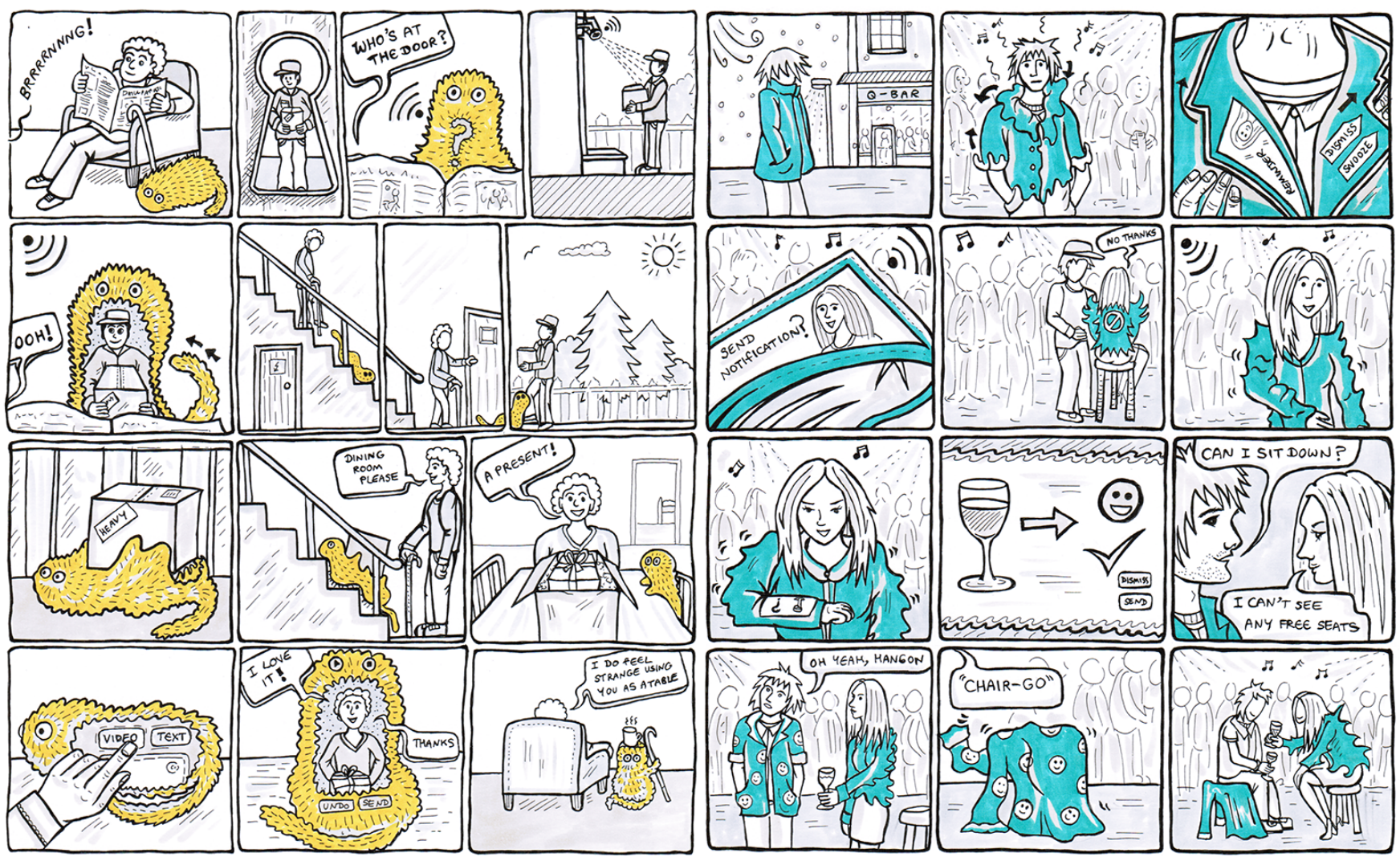

Figure 6: Scenarios based upon authors' Future Work sections in published papers [67]: Left - A robotic pet as home assistant for the infirm [54]; Right - Smart jacket made with variable stiffness fabric [23]. These scenarios were given to researchers and public participants to illustrate new concepts, but also to get feedback on the implications of such technology on peoples' lives - both good and bad. Colour is used to highlight the object of interest in each case, whereas the style of drawing is clear in order to support comprehension. From this work, both parties were able to make suggestions for future research to consider, e.g. what happens when your home support pet is 'hacked'? Would I want to have a jacket that was difficult to wash?

worlds about a subject area of prototype, and unite disparate views in their application. We propose the act of sketching and drawing in $\mathrm{HCI}$ as having three main foci: A sketch is the act of creation, a worked drawing becomes an object of communication, and both outputs are sources of information via analysis and interpretation. The following paragraphs suggest opportunities and methods for adoption, outlining ideation and subjective inquiry, utilising outputs for communication, and how we analyse our imagery.

\subsection{Creation}

Ideation is the start-point for future thinking, and can be subjective, team based, or used as a method of combined inquiry or co-creation with participants. The sketch, rather than the polished, drawn image lends itself best to this form. Design provides a valuable resource of techniques for inspiring idea generation, such as the De Bono method [19] or design thinking [18] - and many more. However, successful ideation via sketching can take place without the application of design-led techniques [63]. By providing questions or prompts for participants, and a clear direction, ideas can be generated for complex, technical outcomes [62,70]. The important aspects of participant sketching for ideation in HCI remain informational (what background knowledge do your participants need?), material (provide the tools with which to create without boundaries), and encouraging (prompts and reassurance as to the desired quality of the image).

These suggestions can also be used within teams in the workshop or co-creation setting. Following ideation, individuals can be led to elaborate upon their initial, or favourite response, and create drawings that delve deeper into the desired object or story. Where confidence in skill is lacking, the creation of these more detailed images can be a team effort where viable. Figure 2 shows a combined approach of both participant-based ideation and subjective inquiry by the researcher, working through ideas and concepts to address potential problems and outcomes in development of a prototype [67]. Sketching problems can be as valuable as sketching successful outcomes, and can "future-proof" the design and build process. 


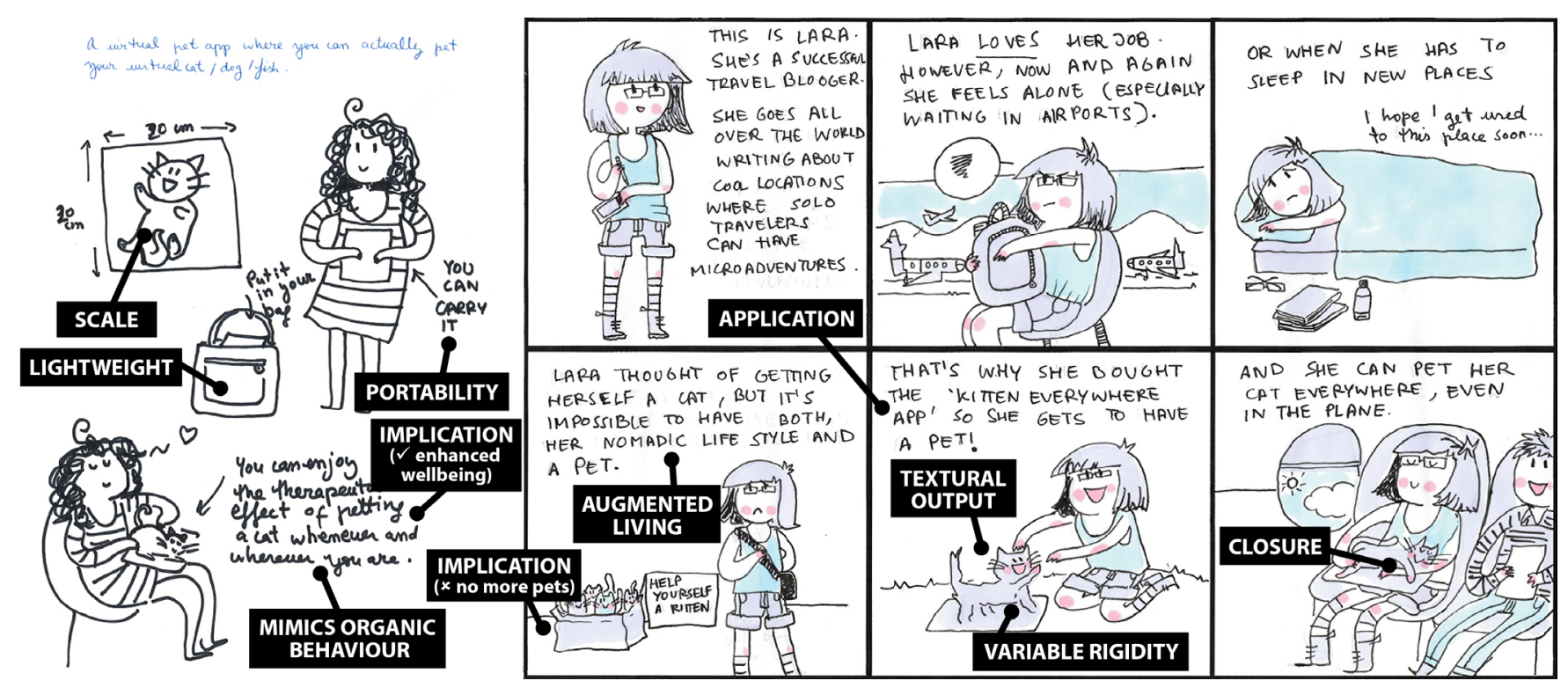

Figure 7: Identifying design requirements and implications from participant imagery [62]. These images were produced by a participant with no prior experience within computer science. They ideated around a concept about loneliness in the digital age, and the transience of modern life not allowing for the acquisition of pets. In the diagram they consider what size a digital, tangible pet would need to be to support travel, and what it might look like. The scenario elaborates upon the features in the diagram, and allows us to look deeper into the more technical requirements of such an application. This work used sketching alongside an open coding approach.

Researcher led sketching can be supported by ongoing learning and development to refine motor-skills and personal style.

\subsection{Communication}

Images created via the ideation process can branch into two outcomes: the elaborative diagram or explanation, to use to communicate concepts and practical aspects of an idea, often internally within teams; and the outward facing image, showing process, containing a story, or existing as an artefact, with an audience beyond the institutional setting. Internally, elaborative drawing can produce blueprints for builds and processes, and remain as a tool that can be referred back to which is easy to understand and interrogate. The elaborative work can also be translated into UML or CAD drawings (for example) where precision lines and relationships are needed for practically engineering a solution. This suggests the need for sketching and drawing for team members at all levels. In the outward facing context, the drawn image becomes an impactful resource, to be utilised in published work to show process and outcome, or as a method of outreach to non-technical audiences. The nature of drawn images for communication suggests moving away from ambiguity, to clear, interpretable outcomes with practical applications, such as scenarios and comics, visual abstracts, or evidence of process and development.

\subsection{Information}

The informational aspects of sketching and drawing can be gleamed from both the outward facing imagery, but also from sketches and elaborative drawings, thus the drawn image becomes an object of analysis. Analysis can be large scale, as in the identification of design patterns, icons, co-occurring motifs (such as for graphical abstracts [33]), or on a case by case basis, as outlined in the elicitation of requirements or meaning through graphical diagramming and storytelling [62]. Images lend themselves to qualitative analysis techniques such as thematic analysis [8], but also to novel, hybridised techniques such as identifying implications of the adoption of our technologies [47, 62]. Analysis of the drawn image can also be in the form of reactive responses, via presentation to participants or stakeholders, for example, the scenarios in Figure 6 were used to explore potential public reactions to the prospect of living with such devices [67]). In essence, once produced, images themselves can become boundary objects for further inquiry.

\section{DISCUSSION}

The works contained within this paper show how we can embrace sketching and drawing for future inquiry in $\mathrm{HCI}$. The provenance and potential of this area is not in doubt, what remains is the question of how we can encourage adoption of these techniques into the wider community in a targeted way to deliver maximum impact and in support of more traditional research approaches? In this respect, the concept of building teaching and research communities around this arts-based approach becomes key: the continued offering of opportunities to engage with students and professionals alike, forums for advice and questions, databases of relevant research, and so forth [64]. The current lack of perceived value, support and training for creative practice within HCI remains the biggest limitation of the approach. Sketching and drawing is often seen as a "soft" skill, 
of lower value than technical practices and outputs such as coding and writing, or a "hobby" - but we show here the added value they can bring to the research table.

Beyond the inherent merit of the examples such approaches also offer timely responses to the increasingly complex demands of our field. HCI's persistent concern with "proximate futures" puts pressure on researchers not only to demonstrate feasibility, but to unpack the implications of innovation [47]. Moreover the movingtarget of disruptive progress, particularly around the Internet of Things (IOT) and so-called Artificial Intelligence (AI), disrupts our well-established, ways of viewing the world and how our research sits within it [56]. These 'shifting sands' of our socio-technical reality demand new ways of looking at it and researching it. Recently, responding to an increasing interest in 'post-anthropocentric' perspectives such as Object-Oriented Ontology [48], Thing Ethnography [27], and Postphenomenology [72], proposals to augment the traditions of Human-Centred Design to become a More-Than Human Centred Design approach, have taken shape [16]. The sketch-based approaches outlined and reviewed in this paper are apt and timely approaches ways to respond to these needs. We are in the midst of a paradigm shift, and while we become accustomed to the new reality, methods which are nimble, efficient and insightful are needed to help us be agile and adapt at a rate traditional approaches are not able to. Sketch-based inquiries can play a part in addressing this clear-and-present need.

Hand-drawn outputs remain a self-contained method of future inquiry, but have the potential to be further expanded by the very technologies that we strive to produce as a community. In the introduction, we suggest ways in which the field embraces and expands upon traditional perspectives of what drawing is, by refining it digitally and breathing new life and direction into a reflex that we, as humans, have had since we first developed motor-skills. We do not focus on the digital/paper divide in the related work, as to draw with stylus rather than pencil is still the same process, although the very existence of the tablet rather than paper can "interrupt" or change the ideation and thinking process in comparative studies [34]. There is no doubt that the hand-drawn sketch can be more than the sum of its parts, but it also exists in a safe space - remaining relevant despite the advances of technology [28].

The "humble line" can therefore co-exist with its future self, where it fuels discovery and outputs, from becoming a skill also belonging to machines [71], to automated mutating from $2 \mathrm{D}$ image to 3D structure [35]. The rise in advances for brain machine interfaces may also signpost a world where the hand no longer conveys the thoughts and ideas of the mind [69]. These types of advances embrace more than human design, our AI capabilities are growing, can we use the humble line to build bridges between the human and machine world, through shared sketching and drawing practice? These practices are simple, as well as material and labour un-intensive, yet have the capacity to represent complexity - for example, could we use sketching to represent bias in AI systems? Could we use emotionally based drawing to inform a machine as to how to make decisions or to represent itself? We wish to start these conversations.

What remains however, is that it all starts with a sketch. The drawn image has no boundaries to expression, and contains worlds of emotion, background, culture, and unknowns. These creative practices underpin a wide swathe of HCI research, but exist as a methodology in their own right, and can support and advance our field as we move through the next century and beyond. We hope that this provocation can start a dialogue and instil the value of traditional, hand-drawn imagery into a highly technological future. Will you be drawn into the discussion?

\section{CONCLUSION}

Sketching and drawing are already methods of future inquiry, but can benefit from greater transparency in research process, communication to diverse groups within HCI, and wider adoption within the community. No other method lends itself to novice and expert alike, is quick and cheap to produce, and can have such a varied influence on a final idea, process or product. Case studies and related work offer evidence of success, and can provide guidelines for those who require assistance in working this artistic practice into their projects. Further, by blending sketching and drawing as technique with prototypical and maker practice, there is the potential for our drawings of the future to quite literally jump off the page as concrete technologies. Drawing is vision.

\section{ACKNOWLEDGMENTS}

Thanks to the Sketching in HCI community, namely Makayla Lewis and Nicolai Marquardt, and all the practitioners that lend their research and practice for the discovery of others in this domain.

\section{REFERENCES}

[1] Alberto Alessi. 2001. The dream factory: Alessi since 1921. Electa/Alessi.

[2] Nancy C Andreasen. 2012. Creativity in art and science: are there two cultures? Dialogues in clinical neuroscience 14, 1 (2012), 49.

[3] Benjamin Bach, Nathalie Henry Riche, Sheelagh Carpendale, and Hanspeter Pfister. 2017. The emerging genre of data comics. IEEE computer graphics and applications 37, 3 (2017), 6-13.

[4] Eli Blevis, Sabrina Hauser, and William Odom. 2015. Sharing the hidden treasure in pictorials. interactions (2015).

[5] Mark Blythe. 2017. Research fiction: storytelling, plot and design. In Proceedings of the 2017 CHI Conference on Human Factors in Computing Systems. ACM, 54005411.

[6] Mark Blythe, Kristina Andersen, Rachel Clarke, and Peter Wright. 2016. Antisolutionist strategies: Seriously silly design fiction. In Proceedings of the 2016 CHI Conference on Human Factors in Computing Systems. ACM, 4968-4978.

[7] Adrien Bousseau, Theophanis Tsandilas, Lora Oehlberg, and Wendy E Mackay. 2016. How novices sketch and prototype hand-fabricated objects. In Proceedings of the 2016 CHI Conference on Human Factors in Computing Systems. ACM, 397-408.

[8] Virginia Braun, Victoria Clarke, Nikki Hayfield, and Gareth Terry. 2019. Thematic analysis. Handbook of Research Methods in Health Social Sciences (2019), 843-860.

[9] Jeffrey Browne, Bongshin Lee, Sheelagh Carpendale, Timothy Sherwood, and Nathalie Riche. 2011. iSketchVis: Integrating Sketch-based Interaction with Computer Supported Data Analysis. GSWC 2011 (2011), 25.

[10] Bill Buxton. 2010. Sketching user experiences: getting the design right and the right design. Morgan kaufmann.

[11] Yang Cao, Hai Wang, Changhu Wang, Zhiwei Li, Liqing Zhang, and Lei Zhang. 2010. Mindfinder: interactive sketch-based image search on millions of images. In Proceedings of the 18th ACM international conference on Multimedia. ACM, 1605-1608.

[12] Qi Chen, John Grundy, and John Hosking. 2003. An e-whiteboard application to support early design-stage sketching of UML diagrams. In IEEE Symposium on Human Centric Computing Languages and Environments, 2003. Proceedings. 2003. IEEE, 219-226.

[13] Eunyoung Chung, Carlos Jensen, Koji Yatani, Victor Kuechler, and Khai N Truong. 2010. Sketching and drawing in the design of open source software. In 2010 IEEE Symposium on Visual Languages and Human-Centric Computing. IEEE, 195-202.

[14] Neil Cohn. 2012. Explaining âĂŸI can't drawâĂŹ: Parallels between the structure and development of language and drawing. Human Development 55, 4 (2012), 167-192. https://doi.org/10.1159/000341842

[15] Paul Coulton, Joseph Lindley, Miriam Sturdee, Mike Stead, et al. 2017. Design fiction as world building. (2017). 
[16] Paul Coulton and Joseph Galen Lindley. 2019. More-Than Human Centred Design: Considering Other Things. The Design fournal (2019), 1-19.

[17] Brock Craft and Paul Cairns. 2009. Sketching sketching: outlines of a collaborative design method. In Proceedings of the 23rd British HCI Group Annual Conference on People and Computers: Celebrating People and Technology. British Computer Society, 65-72.

[18] Alma L Culén and Asbjørn Følstad. 2014. Innovation in HCI: what can we learn from design thinking?. In Proceedings of the 8th Nordic Conference on HumanComputer Interaction: Fun, Fast, Foundational. ACM, 849-852.

[19] Edward De Bono. 1986. De Bono's thinking course. (1986).

[20] Tomás Dorta. 2008. Design flow and ideation. International fournal of Architectural Computing 6, 3 (2008), 299-316.

[21] Thomas Dykes, Mark Blythe, Jayne Wallace, James Thomas, and Tim Regan. 2016 RtD comics: A medium for representing research through design. In Proceedings of the 2016 ACM Conference on Designing Interactive Systems. ACM, 971-982.

[22] Stephanie Foehrenbach. 2015. Learn to Sketch (Even if You Can't Draw): Handson Sketching Course. In Proceedings of the 33rd Annual ACM Conference Extended Abstracts on Human Factors in Computing Systems. ACM, 2467-2468.

[23] Sean Follmer, Daniel Leithinger, Alex Olwal, Nadia Cheng, and Hiroshi Ishii. 2012. Jamming user interfaces: programmable particle stiffness and sensing for malleable and shape-changing devices. In Proceedings of the 25th annual ACM symposium on User interface software and technology. ACM, 519-528.

[24] Christopher Frayling. 1993. Research in art and design. (1993).

[25] Kevin Gallagher, Sameer Patil, and Nasir Memon. 2017. New me: Understanding expert and non-expert perceptions and usage of the Tor anonymity network. In Thirteenth Symposium on Usable Privacy and Security (\{SOUPS \} 2017). 385-398.

[26] William W Gaver, Jacob Beaver, and Steve Benford. 2003. Ambiguity as a resource for design. In Proceedings of the SIGCHI conference on Human factors in computing systems. ACM, 233-240.

[27] Elisa Giaccardi, Nazli Cila, Chris Speed, and Melissa Caldwell. 2016. Thing ethnography: doing design research with non-humans. In Proceedings of the 2016 ACM Conference on Designing Interactive Systems. ACM, 377-387.

[28] Gabriela Goldschmidt. 2017. Manual sketching: Why is it still relevant? In The Active Image. Springer, 77-97.

[29] Simon Grand and Martin Wiedmer. 2010. Design fiction: a method toolbox for design research in a complex world. In proceedings of the DRS 2010 conference: Design and Complexity.

[30] Saul Greenberg, Sheelagh Carpendale, Nicolai Marquardt, and Bill Buxton. 2011 Sketching user experiences: The workbook. Elsevier.

[31] Mieke Haesen, Kris Luyten, and Karin Coninx. 2009. Get your requirements straight: Storyboarding revisited. Human-Computer Interaction-INTERACT 2009 (2009), 546-549.

[32] Tracy Hammond and Paul Taele. 2019. Sketching Cognition and Creativity: Leveraging Sketch Interfaces for Enhancing Creativity and Cognition. In Proceedings of the 2019 on Creativity and Cognition. ACM, 708-713.

[33] Jessica Hullman and Benjamin Bach. 2018. Picturing Science: Design Patterns in Graphical Abstracts. In International Conference on Theory and Application of Diagrams. Springer, 183-200.

[34] Rahinah Ibrahim and Farzad Pour Rahimian. 2010. Comparison of CAD and man ual sketching tools for teaching architectural design. Automation in Construction 19, 8 (2010), 978-987.

[35] Takeo Igarashi, Takeo Igarashi, Satoshi Matsuoka, and Hidehiko Tanaka. 2007 Teddy: a sketching interface for 3D freeform design. In Acm siggraph 2007 courses ACM, 21.

[36] Brian David Johnson. 2011. Science fiction prototyping: Designing the future with science fiction. Synthesis Lectures on Computer Science 3, 1 (2011), 1-190.

[37] Gabe Johnson, Mark Gross, Ellen Yi-Luen Do, and Jason Hong. 2012. Sketch it, make it: sketching precise drawings for laser cutting. In CHI'12 Extended Abstracts on Human Factors in Computing Systems. ACM, 1079-1082.

[38] Gabe Johnson, Mark D Gross, Jason Hong, Ellen Yi-Luen Do, et al. 2009. Computational support for sketching in design: a review. Foundations and Trends ${ }^{\circledR}$ in Human-Computer Interaction 2, 1 (2009), 1-93.

[39] Ben Jonson. 2005. Design ideation: the conceptual sketch in the digital age Design studies 26, 6 (2005), 613-624.

[40] James A Landay and Brad A Myers. 1996. Sketching storyboards to illustrate interface behaviors. In Conference on Human Factors in Computing Systems: Conference companion on Human factors in computing systems: common ground, Vol. 13 193-194.

[41] Makayla Lewis and Lizzie Coles-Kemp. 2014. A Tactile Visual Library To Support User Experience Storytelling. In Proceedings of NordDesign. 386.

[42] Makayla Lewis, Miriam Sturdee, Jason Alexander, Jelle Van Dijk, Majken Kirkegård Rasmussen, and Thuong Hoang. 2017. SketchingDIS: Handdrawn Sketching in HCI. In Proceedings of the 2016 ACM Conference Companion Publication on Designing Interactive Systems. ACM, 356-359.

[43] Makayla Lewis, Miriam Sturdee, and Nicolai Marquardt. 2018. Applied Sketching in HCI: Hands-on Course of Sketching Techniques. In Extended Abstracts of the 2018 CHI Conference on Human Factors in Computing Systems. ACM, C08.
[44] Makayla Lewis, Miriam Sturdee, and Nicolai Marquardt. 2019. Sketching in HCI: Hands-on Course of Sketching Techniques. In Extended Abstracts of the 2019 CHI Conference on Human Factors in Computing Systems. ACM, C04.

[45] Makayla Lewis, Miriam Sturdee, Nicolai Marquardt, and Thuong Hoang. 2018. SketCHI: Hands-On Special Interest Group on Sketching in HCI. In Extended Abstracts of the 2018 CHI Conference on Human Factors in Computing Systems (CHI EA '18). ACM. https://doi.org/10.1145/3170427.3185366

[46] Makayla Lewis, Miriam Sturdee, Jagoda Walny, Nicolai Marquardt, Thuong Hoang, Joanna Foster, and Sheelagh Carpendale. 2019. SketCHI 2.0: HandsOn Special Interest Group on Sketching in HCI. In Extended Abstracts of the 2019 CHI Conference on Human Factors in Computing Systems. ACM, SIG12.

[47] Joseph Lindley, Paul Coulton, and Miriam Sturdee. 2017. Implications for adoption. In Proceedings of the 2017 CHI Conference on Human Factors in Computing Systems. ACM, 265-277.

[48] Joseph Galen Lindley, Paul Coulton, and Haider Akmal. 2018. Turning Philosophy with a Speculative Lathe: Object Oriented Ontology, Carpentry, and Design Fiction. (2018)

[49] Conor Linehan, Ben J Kirman, Stuart Reeves, Mark A Blythe, Joshua G Tanenbaum, Audrey Desjardins, and Ron Wakkary. 2014. Alternate endings: using fiction to explore design futures. In CHI'14 Extended Abstracts on Human Factors in Computing Systems. ACM, 45-48.

[50] Andrew Loomis. 1963. Successful Drawing. Three-Dimensional Drawing.(Revised Edition of Successful Drawing.) With Illustrations. Chapman \& Hall.

[51] Aaron Marcus. 2015. HCI goes mainstream in the comics. In HCI and UserExperience Design. Springer, 265-269.

[52] Nicolai Marquardt and Saul Greenberg. 2012. Sketchnotes for Visual Thinking in HCI. In Proc. ACM Conference on Human Factors in Computing Systems: CHI Workshop on Visual Thinking and Digital Imagery.(Workshop held at the ACM CHI Conference), Vol. 5.

[53] Francis C Moon. 2007. The Machines of Leonardo Da Vinci and Franz Reuleaux: kinematics of machines from the Renaissance to the 20th Century. Vol. 2. Springer Science \& Business Media.

[54] Takuya Nojima, Yoshiharu Ooide, and Hiroki Kawaguchi. 2013. Hairlytop interface: An interactive surface display comprised of hair-like soft actuators. In 2013 World Haptics Conference (WHC). IEEE, 431-435.

[55] Mizuki Oka, Kazuhiko Kato, Yingqing Xu, Lin Liang, and Fang Wen. 2008. Scribblea-secret: Similarity-based password authentication using sketches. In 2008 19th International Conference on Pattern Recognition. IEEE, 1-4.

[56] James Pierce and Carl DiSalvo. 2017. Dark Clouds, Io\&\#!+, and [Crystal Ball Emoji]: Projecting Network Anxieties with Alternative Design Metaphors. In Proceedings of the 2017 Conference on Designing Interactive Systems. ACM, 13831393.

[57] Majken Kirkegård Rasmussen, Giovanni Maria Troiano, Marianne Graves Petersen, Jakob Grue Simonsen, and Kasper Hornbæk. 2016. Sketching Shapechanging Interfaces: Exploring Vocabulary, Metaphors Use, and Affordances.. In CHI. 2740-2751.

[58] Pieter Jan Stappers and James M Hennessey. 1999. Toward electronic napkins and beermats: Computer support for visual ideation skills. In Visual representations and interpretations. Springer, 220-225.

[59] Miriam Sturdee, Jason Alexander, Paul Coulton, and Sheelagh Carpendale. 2018. Sketch \& The Lizard King: Supporting image inclusion in HCI publishing. In Extended Abstracts of the 2018 CHI Conference on Human Factors in Computing Systems. ACM, alt15.

[60] Miriam Sturdee, Paul Coulton, and Jason Alexander. 2017. Using Design Fiction to Inform Shape-Changing Interface Design and Use. The Design fournal 20, sup1 (2017), S4146-S4157.

[61] Miriam Sturdee, Paul Coulton, Joseph G Lindley, Mike Stead, Haider Ali, and Andy Hudson-Smith. 2016. Design fiction: How to build a Voight-Kampff machine. In Proceedings of the 2016 CHI Conference Extended Abstracts on Human Factors in Computing Systems. ACM, 375-386.

[62] Miriam Sturdee, Aluna Everitt, Joseph Lindley, Paul Coulton, and Jason Alexander. 2019. Visual Methods for the Design of Shape-Changing Interfaces. In IFIP Conference on Human-Computer Interaction. Springer, $\mathrm{xx}-\mathrm{xx}$.

[63] Miriam Sturdee, John Hardy, Nick Dunn, and Jason Alexander. 2015. A Public Ideation of Shape-Changing Applications. In Proceedings of the 2015 International Conference on Interactive Tabletops \& Surfaces. ACM, 219-228.

[64] Miriam Sturdee, Makayla Lewis, and Nicolai Marquardt. 2018. Feeling SketCHI?: the lasting appeal of the drawn image in HCI. interactions 25, 6 (2018), 64-69.

[65] Miriam Sturdee, Makayla Lewis, and Nicolai Marquardt. 2018. SketchBlog\# 1: the rise and rise of the sketchnote. interactions 25, 6 (2018), 6-8.

[66] Miriam Sturdee, Samuel Mann, and Sheelagh Carpendale. 2019. Sketching Sustainability in Computing. In Proceedings of the 2019 on Creativity and Cognition. ACM, 29-40.

[67] Miriam Amber Sturdee. 2018. Sketching as a support mechanism for the design and development of shape-changing interfaces. Ph.D. Dissertation. Lancaster University.

[68] Paul Taele, Laura Barreto, and Tracy Hammond. 2015. Maestoso: an intelligent educational sketching tool for learning music theory. In Twenty-Seventh IAAI 
Conference.

[69] Desney Tan and Anton Nijholt. 2010. Brain-computer interfaces and humancomputer interaction. In Brain-Computer Interfaces. Springer, 3-19.

[70] Maryam Tohidi, William Buxton, Ronald Baecker, and Abigail Sellen. 2006. User sketches: a quick, inexpensive, and effective way to elicit more reflective user feedback. In Proceedings of the 4th Nordic conference on Human-computer interaction: changing roles. ACM, 105-114.

[71] Patrick Tresset and Frederic Fol Leymarie. 2013. Portrait drawing by Paul the robot. Computers \& Graphics 37, 5 (2013), 348-363.

[72] Ron Wakkary, Doenja Oogjes, Sabrina Hauser, Henry WJ Lin, Cheng Cao, Leo Ma, and Tijs Duel. 2017. Morse Things: A Design Inquiry into the Gap Between Things and Us.. In Conference on Designing Interactive Systems. 503-514.

[73] Jagoda Walny, Jonathan Haber, Marian Dörk, Jonathan Sillito, and Sheelagh Carpendale. 2011. Follow that sketch: Lifecycles of diagrams and sketches in software development. In Visualizing Software for Understanding and Analysis (VISSOFT), 2011 6th IEEE International Workshop on. IEEE, 1-8.

[74] Blake Williford. 2017. SketchTivity: Improving Creativity by Learning Sketching with an Intelligent Tutoring System. In Proceedings of the 2017 ACM SIGCHI Conference on Creativity and Cognition. ACM, 477-483.

[75] Dustin Wüest, Norbert Seyff, and Martin Glinz. 2015. Sketching and notation creation with FlexiSketch Team: Evaluating a new means for collaborative requirements elicitation. In 2015 IEEE 23rd International Requirements Engineering Conference (RE). IEEE, 186-195.

[76] Sarita Yardi, Pamela Krolikowski, Taneshia Marshall, and Amy Bruckman. 2008. An HCI approach to computing in the real world. fournal on Educational Resources in Computing (fERIC) 8, 3 (2008), 9. 